\title{
Áreas en riesgo de invasión por Procambarus clarkii (Decapoda: Cambaridae) un cangrejo de río introducido en Colombia
}

\author{
Rosa F. Camacho-Portocarrero ${ }^{1}$, Irene Duarte-Gándica ${ }^{1,2}$ \& Mariano Altamiranda-Saavedra ${ }^{3 *}$ \\ 1. Facultad de Ciencias Básicas y Tecnologías, Universidad del Quindío Armenia, Quindío Colombia; \\ rfcamachop@uqvirtual.edu.co \\ 2. Escuela de Investigación en Biomatemáticas, Universidad del Quindío, Armenia, Quindío, Colombia; \\ iduarte@uniquindio.edu.co \\ 3. Grupo de Investigación Bioforense, Facultad de Derecho y Ciencias Forenses, Tecnológico de Antioquia Institución \\ Universitaria, Medellín, Colombia; maltamiranda2@gmail.com \\ * Correspondencia
}

Recibido 21-IV-2020. Corregido 12-X-2020. Aceptado 21-X-2020.

\begin{abstract}
Areas at risk of invasion by Procambarus clarkii (Decapoda: Cambaridae) a crayfish introduced in Colombia. Introduction: Biological invasions are a major threat to biodiversity and ecosystems, with current and potential impacts on public health and conservation. Procambarus clarkii is a decapod crustacean, native to the Southern United States and Northeastern Mexico, which may adapt to different environmental conditions due to its ecological plasticity. Objective: The existing fundamental ecological niche of Procambarus clarkii was characterized in order to predict areas with environmental suitability for the potential establishment of the species in South America and Colombia. Methods: We used models of calibrated ecological niches in the native area, elaborated with the Maxent algorithm, based on occurrence data extracted from GBIF and hydroclimatic variables of aquatic ecosystems at a resolution of $1 \mathrm{~km}^{2}$. Results: The model indicated a wide geographic area for invasion potential in South America, predicting environmentally suitable areas for the presence and expansion towards Colombia, Venezuela, Peru, Ecuador, Brazil, Guyana, Surinam, Bolivia, Uruguay, Argentina, Paraguay and Chile. In Colombia, suitable areas predicted by the model are located mainly in the North and East of the country, spanning a diversity of ecosystems, such as tropical forests, basal forests, riparian forests and savannas. The species has a high possibility of expanding into low latitude distribution areas, occupying areas towards the Northern part of the Colombian Caribbean region, in the departments of Magdalena, Cesar, Cordoba and Atlántico. Suitability areas environmental were also predicted in Eastern Colombia, towards the extension of the Eastern plain of the Orinoquia, a low altitude region in Arauca, Casanare, Meta and Vichada. Conclusion: This study applies ecological niche models, which may be of interest in the planning of strategies or the creation of management plans, such as early warning systems that prevent the establishment of this species.
\end{abstract}

Key words: red swamp crayfish; management exotic species; ecological niche; potential distribution.

Camacho-Portocarrero, R.F., Duarte-Gándica, I., \& Altamiranda-Saavedra, M. (2021). Áreas en riesgo de invasión por Procambarus clarkii (Decapoda: Cambaridae) un cangrejo de río introducido en Colombia. Revista de Biología Tropical, 69(1), 77-89. DOI $10.15517 /$ rbt.v69i1.41493

Las especies invasoras representan un agente significativo de cambio global (Vitousek, D’Antonio, Lloyd, \& Westbrooks, 1996) con implicaciones económicas y ambientales potencialmente graves, generando efectos negativos en la agricultura, la salud pública, el transporte y la conservación (Pimentel, Lach, Zuniga, \& Morrison 2000; Sousa, Morais, Dias, \& Antunes, 2011). Después de la destrucción de hábitat, la invasión de especies exóticas es el segundo factor asociado a la extinción de especies en el mundo (Everett, 2000). 
De hecho, como las especies invasoras se extienden a hábitats naturales, pueden afectar áreas amplias, por lo que, la capacidad de predecir y anticipar su potencial de dispersión geográfica es indispensable para diseñar programas de control preventivos (Simberloff, Parker, \& Windle, 2005). Dada la importancia de comprender las complejidades de las dinámicas de invasión en aplicaciones reales, se necesita de nuevas metodologías para predecir las invasiones de especies (Sakai et al., 2001; Gallien, Münkemüller, Albert, Boulangeat, \& Thuiller, 2010). Recientemente, el marco predictivo que se ha aplicado en materia de especies invasoras, son los modelos de nicho ecológico (MNE) (Peterson, 2003). El enfoque de estos modelos permite a los investigadores caracterizar regiones en las que encuentran los requerimientos para la presencia de las especies (Peterson et al., 2011).

El uso de modelos correlativos para estimar la distribución de especies es cada vez más factible, en parte, debido al aumento en la disponibilidad de datos climáticos y ambientales de alta resolución y los avances en los métodos de modelado. Estos modelos identifican relaciones estadísticas entre ubicaciones de presencia de especies y un conjunto de predictores ambientales en el espacio geográfico, que luego se utilizan para identificar otras áreas con condiciones ambientales similares haciendo estimaciones de idoneidad relativa (Elith, 2013). La simplicidad de la entrada de datos hace que los modelos correlativos sean particularmente atractivos para estimar hábitats idóneos para especies invasoras. Sin embargo, la extrapolación de las predicciones a nuevas áreas que son de interés, en la mayoría de aplicaciones a especies invasores, requiere de precaución en parte por los efectos geográficos o acceso a todo el conjunto de condiciones que la especie puede tolerar, a la sensibilidad de la información con la que se cuenta y la capacidad de dispersión o de acceso a las condiciones de nicho (Thuiller et al., 2005; Elith, 2013).

Para el presente estudio, se consideró como organismo modelo, el cangrejo de río Procambarus clarkii (Girard, 1852), una especie invasora, originaria del noreste de México y centro-sur de los Estados Unidos (Hobbs, 1972). Esta especie, se ha establecido en casi todos los continentes por vía antrópica, excepto en la Antártida y Australia (Huner, 1977; Huner \& Avault, 1979), como una especie de importancia económica para la acuicultura y comercio ornamental (Barbaresi, Santini, Tricarico, \& Gherardi, 2004). Pero una vez que ha sido introducida naturalmente, se descarta una vía de dispersión natural contigua en los ríos. Análisis genéticos han revelado evidencia de múltiples introducciones mediadas por humanos en China en la cuenca del río Pearl y cuencas hídricas adyacentes. Las actividades como el transporte comercial fueron probablemente las responsables de la dispersión a larga distancia de P. clarkii (Huang, Tang, Cai, Lin, \& Wu, 2017).

Esta especie posee estrategia $r$, lo que le ha merecido ser considerada como una de las especies con mayor plasticidad ecológica entre los decápodos (Campos, 2005). Es responsable de grandes modificaciones en ambientes invadidos, causando daños ecológicos y económicos considerables. En el 2017, la producción de $P$. clarkii superó el millón de toneladas para un impacto económico total de más de $38.6 \mathrm{mil}$ millones de dólares en China (Yi et al., 2018). Esta especie tiene amplia tolerancia ambiental a una variedad de condiciones ambientales, incluida la mala calidad del agua, las fluctuaciones de temperatura y las bajas concentraciones de oxígeno. La rápida tasa de crecimiento poblacional, cuidado parental y competitividad por recursos, causa fuertes presiones sobre los ecosistemas (Gherardi \& Acquistapace, 2007). La excavación de madrigueras en épocas de mudas y posturas, provoca daños en diques, represas y en actividades agrícolas de áreas inundadas como plantaciones de arroz (Barbaresi et al., 2004). Los impactos documentados se centran en los efectos de disminución de macrófitas y depredación directa de huevos, larvas, juveniles o adultos en varias especies (anfibios, moluscos y macroinvertebrados) mostrando como esta pérdida de biodiversidad conduce a un desequilibrio de la dinámica de 
la red trófica en ecosistemas invadidos (Cruz \& Rebelo, 2005; Souty-Grosset et al., 2016). Además $P$. clarkii es vector del hongo Aphanomyces astaci y propaga enfermedades a una parte de la fauna nativa como ha ocurrido con Austropotamobius pallipes (Aquiloni, Martin, Gherardi, \& Diéguez-Uribeondo, 2011). Por lo tanto, el manejo oportuno de la invasión de esta especie es necesario para mitigar los posibles impactos negativos ya mencionados.

Diversas investigaciones sobre $P$. clarkii, a nivel mundial y de Suramérica se han enfocado en investigar aspectos biológicos, reproductivos, morfológicos, fisiológicos, genéticos y ecológicos de la especie, que influyen en la dinámica de invasión, (Romero-Trigueros, 1998; Torres \& Álvarez, 2012; Loureiro, Anastácio, Araujo, Souty-Grosset, \& Almerão, 2015; Pedroza-Martínez, 2017; Loureiro, Anastácio, Bueno, \& Araujo, 2018; Gál et al., 2018; Pachón \& Valderrama, 2018). A excepción de un estudio de conservadurismo de nicho de la especie, realizado por Palaoro, Dalosto, Costa, \& Santos, (2013) en el que comunica que $P$. clarkii conserva su nicho en las regiones invadidas y potenciales a invasión en áreas grandes de Suramérica aparentemente adecuadas, principalmente en: Argentina, Chile, Paraguay, Uruguay y sureste de Brasil. Franco (2014), realizó un estudio de morfometría, distribución actual y potencial en el norte de México, en el que los resultados mostraron que la especie actualmente se distribuye en 12 regiones hidrológicas del norte de México, a través de diversos cuerpos de agua dulce, entre ellos: ríos, arroyos, canales de riego, estanques y represas.

Para Suramérica, se introdujo en la región costera de Taura en Ecuador, en el período 1986-1988 con fines de acuicultura (Mora, Uyuguari, \& Osorio, 2004). Se inició un proyecto de "cultivo mixto" en Guayaquil, similar a Luisiana (EE. UU), donde se cultivaba $P$. clarkii en rotación con arroz (Chien \& Avault, 1983; Halwart \& Gupta, 2004). Sin embargo, el proyecto fue abandonado porque la producción de $P$. clarkii no era rentable. La especie probablemente escapó cuando vendedores locales la llevaron a Imbabura, o quizás fue introducida intencionalmente como fuente de alimento (Mora et al., 2004). Rodríguez y Suárez (2001) registran la introducción de la especie en Venezuela, especialmente con fines ornamentales. Magalhães et al., (2005) expresaron que $P$. clarkii se había vendido en tiendas de acuarios desde 1985, lo que sugiere que el comercio de mascotas podría ser el principal vector de introducción en Brasil, pero no realizaron una investigación sistemática. En otras regiones del continente americano, se reconoce la presencia en México, Chile, Belice, Costa Rica, República Dominicana, Nicaragua y Guatemala, pero no hay reportes de información sobre las vías de introducción e historias de invasión (Crandall, 2010). En la actualidad se conoce de poblaciones establecidas en Ecuador, Brasil y Colombia (Pedroza-Martinez, 2017; Riascos et al., 2018; Loureiro et al., 2018).

De acuerdo a los registros, en Colombia fue introducida en 1985 desde EE. UU, como especie experimental para fines comerciales en el Valle del Cauca (Gutiérrez, Lasso, Baptiste, Sánchez-Duarte, \& Díaz, 2012). Sin embargo, en 1988 después de presentarse una fuga accidental de individuos, estos se dispersaron por medios naturales en el municipio de Palmira y luego por vía antrópica, en Santiago de Cali, Jamundí, Yotoco, Guacarí y Buga drenados por el río Cauca (Flórez-Brand \& EspinozaBeltrán, 2011; Gómez, 2014). En el 2004 ya se registraba para la sabana de Bogotá (Campos, 2005) y desde el 2008 en la laguna Fúquene y el área de influencia del río Suárez (Gutiérrez et al., 2012, Pachón \& Valderrama, 2018). Arias-Pineda $(2012,2018)$ da a conocer registros de la expansión de P. clarkii para Bogotá relacionado con actividades humanas en cinco localidades, entre ellas los humedales Juan Amarillo y Jaboque por liberación accidental.

Flórez-Brand y Espinoza-Beltrán (2011), mencionan que el avance de dispersión de la especie es preocupante, porque ocurre de manera acelerada (mediada por humanos, incluido el transporte intencional o no intencional, combinado a su expansión natural). Se resalta los daños causados, por la construcción 
de madrigueras en los diques de contención en las orillas del río cauca y el establecimiento en zonas de cultivos, afectando de gran manera la producción agrícola, como lo señala Loureiro et al., 2015. El presente estudio, es de los primeros que planea detectar áreas potencialmente idóneas para la especie $P$. clarkii en Colombia, usando como herramienta los modelos de nicho ecológico. El objetivo fue caracterizar el nicho ecológico fundamental existente de $P$. clarkii con preguntas como ¿Cuáles son las áreas con idoneidad ambiental potencial para ser invadidas por P. clarkii en Suramerica y Colombia?, en conjunto con las variables ambientales asociadas a su distribución potencial y así detectar áreas vulnerables de invasión. Esta información puede constituir la base para el diseño de estrategias en programas de control preventivo o sistemas de alerta temprana que eviten el posible establecimiento de esta especie.

\section{MATERIALES Y MÉTODOS}

Registros de presencias: Los registros de presencia se obtuvieron del portal de libre acceso a nivel global (GBIF: www.gbif.org). Los datos se verificaron cuidadosamente para detectar repeticiones y posibles errores en la información de ubicación. Se seleccionaron los registros de la especie de donde es nativa e invasora, eliminando los duplicados y definiendo únicamente los rangos nativos propuestos por Torres y Álvarez (2012). Luego, un polígono (Shapefile) del área nativa de la especie, fue utilizado como mascara para eliminar las presencias que estuvieron por fuera del rango nativo, para esto se utilizó la herramienta ArcGIS 10.3 (ESRI Corporation, Redlands, CA), se creó una base de datos que quedó reducida a 614 registros que constituyeron las muestras de presencias para realizar el modelo.

Datos ambientales: Se utilizaron las 19 variables hidroclimáticas (Freshwater environmental variables) disponibles de EarthEnv versión 1.0 (Domisch, Amatulli, \& Jetz, 2015). Este conjunto de datos se creó siguiendo el marco "bioclim" (consultar http://worldclim. org/bioclim para más detalles), utilizando métricas como la temperatura upstream (promedio o promedio ponderado) y la precipitación upstream (suma o suma ponderada) (Domisch et al., 2015). Estas capas son espacialmente continuas, casi globales y específicas de agua dulce, cada una de las subcuencas de las celdas de la cuadrícula se encuentran a lo largo de la red fluvial HydroSHEDS a un tamaño de resolución de celda de 30 arco-segundos ( $\sim 1 \mathrm{~km}$ en el Ecuador) en el sistema de coordenadas WGS84. Se descargaron utilizando el formato netCDF-4 y se procesaron usando el software R (Development Core Team, 2014).

Región de calibración del modelo (M): La selección del área de calibración constituye un elemento clave en el desarrollo de modelos de nichos ecológicos, que corresponde a un área hipotética que ha sido accesible para la especie (M) (Barve et al., 2011). Definimos esta área como la capacidad de dispersión natural de la especie, reportada en literatura (17 km) (Gherardi \& Barbaresi, 2000). A partir de esta información, usamos esta distancia para generar buffers de $17 \mathrm{~km}$ alrededor de los puntos de presencia conocidos de la especie, utilizando ArcGIS 10.3. (Apéndice Digital 1). Cortamos las capas hidroclimáticas como área de calibración o el M.

Proceso de selección de variables: Se evaluó el grado de correlación de las variables, a través de una matriz de correlación de Pearson, utilizando el programa ArcGIS 10.3. Se eliminaron aquellas variables que presentaron una correlación $(r>0.80)$, y generamos un conjunto de variables para modelar el nicho ecológico de la especie. Si se correlacionaban dos variables $(R \geq 0.8)$, se prefería una variable con mayor sentido biológico según lo reportado en literatura y que contribuyera mayormente al modelo sobre la otra. El grado de contribución individual de las variables al modelo, se evaluó mediante el análisis de Jackknife en el programa Maxent utilizando todas las 19 variables. El conjunto de variables seleccionadas bajo los 
criterios anteriormente mencionados se describe en el (Apéndice Digital 2).

Selección de parámetros óptimos para el modelado: Se utilizó el programa Maxent 3.3.3 para desarrollar el modelo. En vista de la sensibilidad del algoritmo a los ajustes de parámetros particulares y al buen desempeño que ha tenido en análisis que incluyen invasiones de especies (West, Kumar, Brown, Stohlgren, \& Bromberg, 2016). Los parámetros de configuración de los modelos fueron seleccionados utilizando el paquete ENMeval de R (R Development Core Team, 2014). La evaluación de las configuraciones para la creación del modelo de esta especie, se realizó a través de la comparación de los desempeños de 48 modelos de prueba (Muscarella et al., 2014). En éstos, se empleó el método de bloque o "Block" para la partición de los datos de entrenamiento y prueba; ocho valores del parámetro multiplicador de regularización (con intervalos de 0.5 a 4 e incrementos de 0.5 ) y seis tipos de clases, con todas las posibles combinaciones de $\mathrm{L}, \mathrm{LQ}, \mathrm{H}$, LQH, LQHP, y LQHPT (donde L = Lineal, Q $=$ Cuadrática, $\mathrm{H}=$ bisagra, $\mathrm{P}=$ producto, $\mathrm{T}=$ umbral). El método de bloque fue seleccionado debido a que puede ser más apropiado cuando se requiere transferir modelos en espacio y tiempo (Muscarella et al., 2014). Se seleccionó en conjunto, los parámetros finales LQHP, el multiplicador de regularización 1.5 y el valor corregido del delta AICc más bajo (0) para pequeños tamaños de muestras, de acuerdo a los resultados de la prueba. Se utilizaron 10000 puntos como background, siendo este un parámetro por defecto del programa Maxent.

Modelado de nicho y evaluación estadística del modelo: Se evaluó el rendimiento estadístico del modelo, ejecutando 10 réplicas, cada una de las réplicas le calculamos la métrica de ROC parcial (tasa de omisión) implementada por Peterson, Papeş y Soberón (2008) en la interfaz gráfica de Nichetoolbox, permitiendo comparar el rendimiento de cada modelo frente a las expectativas aleatorias. Se utilizó el $70 \%$ de los registros de presencias como datos de calibración y el $30 \%$ restante se utilizaron como datos independientes para la validación estadística. Un umbral (threshold) fue establecido para convertir los resultados crudos de la salida del modelo continuo, en hipótesis binarias de presencias frente a ausencias, utilizando una modificación del enfoque del umbral de reclasificación de presencia de entrenamiento (LTPT) más bajo (Pearson, Raxworthy, Nakamura, \& Peterson, 2007). Éste, tiene en cuenta la posible existencia de algún error en nuestros datos de presencia (y su relación con los conjuntos de datos ambientales, que también pueden contener errores), bajo una tasa de error admisible de $\mathrm{E}=5 \%$. Se identificó el valor del umbral de idoneidad mínimo de los datos de calibración de 0.185 y reclasificamos el mapa de salida logística a un mapa binario. Lo que quiere decir que pixeles por encima de ese valor los toma como 1 o lugares con idoneidad ambiental y pixeles por debajo del umbral los clasificamos como 0 o no idoneidad. Esta modificación del enfoque LTPT evita expandir el área identificada como adecuada artificialmente debido al error en los datos de entrada (Peterson, 2014).

El modelo final calibrado en el $\mathrm{M}$, se proyectó Sur América y luego extrajo la ventana que corresponde a la división política de Colombia, donde la especie se está extendiendo y se alerta de su actividad como invasora, obteniendo así un modelo de nicho ecológico representado geográficamente como un mapa de idoneidad de hábitat de las condiciones hidroclimáticas actuales para P. clarkii.

\section{RESULTADOS}

Los modelos se desempeñaron mejor que las expectativas aleatorias cuando se probaron contra los puntos de ocurrencias independientes, con todos los valores de AUC ratio de parcial ROC superiores a 1.0 y todos los valores de $\mathrm{P}$ inferiores a 0.002 en la validación estadística. El resultado de la réplica 4 tuvo un mejor ajuste en el análisis de ROC parcial, con un valor medio de 1.509738 y $\mathrm{P}<0$ (Apéndice Digital 3). El modelo de nicho ecológico se usó para 
evaluar las áreas potenciales de distribución en América del Sur y Colombia (Apéndice Digital 4 y Apéndice Digital 5). Por otro lado, las variables de mayor contribución al modelo fueron estacionalidad de precipitación río arriba (35.9 $\%$ ), precipitación del mes más lluvioso río arriba (20.2\%), temperatura mínima del mes más frío río arriba (8.5\%), temperatura media anual río arriba $(7.8 \%)$, precipitación del trimestre más húmedo río arriba (6.6\%), temperatura media del trimestre más seco río arriba (5.6\%) y temperatura máxima del mes más cálido río arriba (5.2\%) (Apéndice Digital 2).

En Suramérica el modelo indicó un potencial geográfico amplio de invasión mostrando áreas ambientalmente idóneas para la presencia y expansión hacia Colombia, Venezuela, Perú, Ecuador, Brasil, Guyana, Surinam, Bolivia, Uruguay, Argentina, Paraguay y Chile (Apéndice Digital 4). El modelo también predice idoneidad en el lago Titicaca en la porción compartida entre Perú y Bolivia. Es importante mencionar que la región Amazónica en Ecuador y Perú no fueron identificadas como idóneas hidroclimáticamente.

Para Colombia la distribución potencial de la especie mostró congruencia con la distribución conocida en los departamentos de Boyacá y Cundinamarca, ubicados en la región Andina, contrario al valle del Cauca (Apéndice Digital 5). El modelo muestra, que la especie se extendería hacia áreas de distribución a latitudes más bajas, hacia tierras planas, ocupando zonas de la parte norte de la región Caribe, considerando departamentos como: La Guajira, Magdalena, Cesar, Atlántico, Sucre, Córdoba y Bolívar y hacia la extensión de la planicie oriental de la Orinoquia, una región de baja altitud en: Arauca, Casanare, Meta y Vichada. Además, podría ocupar parte de la Amazonia, en departamentos cercanos a la Orinoquia como: Guainía y Guaviare. Es importante resaltar que el modelo no mostró idoneidad hacia regiones del Sur del Amazonas y la Costa Pacífica.

\section{DISCUSIÓN}

Este estudio, presenta la exploración de una especie acuática como organismo modelo para predecir las áreas con idoneidad ambiental para el potencial establecimiento de $P$. clarkii. Las variables asociadas a la precipitación tienen una alta influencia en la bionomía de los organismos acuáticos, dado que la duración de estaciones secas determina las tasas reproductivas y supervivencia de estas especies (Palaoro et al., 2013). La variable ambiental que tuvo una mayor contribución en el modelo fue la estacionalidad de precipitación. Adicionalmente, las variables de temperatura tienen efectos fisiológicos positivos sobre la tasa de crecimiento y sobre el aumento de la viabilidad reproductiva, influyendo en el metabolismo de P. clarkii en aspectos como la alimentación, muda y eclosión (Palaoro et al., 2013; Loureiro et al., 2015). Arias-Pineda y Rodríguez (2012), destacan que las condiciones ambientales como el clima cálido y el agua bien oxigenada producen efectos positivos en el crecimiento de $P$. clarkii.

En varios continentes $P$. clarkii, es considerada una especie invasora incluyendo Suramérica (Huner, 2002). El modelo predice que en Suramérica se presentan las condiciones ambientales que permiten la invasión para el cangrejo de río, podrían aparecer países como idóneos y no haber sido invadidos. Varios de los países ubicados en la zona ecuatorial, que se muestran como idóneos, no son completamente tropicales, sino que presentan una variedad de condiciones climáticas según la localización orográfica (desde zonas cálidas pueden superar los $20{ }^{\circ} \mathrm{C}$ de temperatura anual, zonas frías de alta montaña, su temperatura anual no supera $\operatorname{los} 10^{\circ} \mathrm{C}$ y las zonas templadas su temperatura anual es de entre 10 y $20^{\circ} \mathrm{C}$ ) (Pourrot, 1983). Además, $P$. clarkii puede adaptarse a diferentes condiciones ambientales debido a rasgos de su historia de vida (como su plasticidad ecológica, crecimiento rápido, madurez temprana, alta tasa de fecundidad, cuidado parental, capacidad 
de adaptar su estrategia reproductiva, período de apareamiento y maduración sexual de acuerdo a las condiciones ambientales) (Sommer, 1984; Alcorlo, Otero, Crehuet, Baltanás, \& Montes, 2006; Loureiro et al., 2015). Mientras que las características biológicas (dispersión, polifagia, capacidad depredadora, competencia por espacio, alimento, flexibilidad en su comportamiento, tolerancia a temperaturas extremas de la superficie, por la construcción de madrigueras que pueden proporcionar un refugio térmico en estos decápodos para evitar la desecación (Atkinson \& Taylor, 1988; Eshky, Atkinson, \& Taylor, 1995). Es lo que permite a esta especie expandir su distribución y convertirse en invasora (Gherardi, 2006). Todos estos factores podrían explicar las amplias zonas de distribución predichas por nuestro modelo para el potencial establecimiento de la especie.

El modelo no dio idóneo en algunas o gran parte de las áreas de Patagonia (pero en algunos lugares aparecen idóneas como en Tierra del Fuego) esto posiblemente se deba a los ambientes templado-fríos de la Patagonia, donde la temperatura media anual es inferior a $10{ }^{\circ} \mathrm{C}$, hasta $3{ }^{\circ} \mathrm{C}$ en la parte más austral y la temperatura mínima que presentan las lagunas templadas es de $2{ }^{\circ} \mathrm{C}$ durante el invierno (Baigún, 2001). Aunque $P$. clarkii puede sobrevivir a una amplia gama de temperaturas, estudios fisiológicos han reportado que los cangrejos de río exhiben muy poca actividad cuando son expuestos a temperaturas de 12 y $16^{\circ} \mathrm{C}$. La falta de actividad del cangrejo de río a bajas temperaturas, puede ser el resultado de una supresión de los procesos metabólicos dependientes de la temperatura, como la frecuencia cardíaca y las tasas de consumo de oxígeno (Eshky et al., 1995, 1996; Stillman \& Somero, 1996; De Pirro, Canicci, \& Santini, 1999).

En tierra de Fuego, el clima de la zona se caracteriza por inviernos y veranos con una temperatura media anual de $5.9^{\circ} \mathrm{C}$, siendo 2.3 y $10{ }^{\circ} \mathrm{C}$ la media de las temperaturas mínimas y máximas (Servicio de Información Ambiental y Geográfica, SIAG, CADIC). Aunque $P$. clarkii sea una especie ectotérmica exitosa, que puede sobrevivir a estas temperaturas, su actividad se ve reducida, por lo que la construcción de madrigueras, no los expone a duras condiciones ambientales para sobrevivir (Chung, Cooper, Graff, \& Cooper, 2012). En tierra del fuego (isla grande), ya se han presentado introducciones de especies exóticas donde, cerca del $30 \%$ de los cauces de la zona andina fueron ocupados por el castor americano en 20 años (Lizarralde, 1993; Schiavini et al., 2016).

Nuestros resultados tienen concordancia con la distribución previamente reportada para esta especie en Brasil, donde se ha evidenciado que tiene 17 poblaciones establecidas, todas ellas en el Sudeste (Loureiro et al., 2018). La llegada a este territorio se atribuye a su introducción para el comercio de acuarios y posteriormente fueron puestos en libertad en la naturaleza, accidental o deliberadamente (Magalhães et al., 2005; Banci, Tollero-Viera, Marinho, Calixto, \& Marques, 2013; Loureiro et al., 2018). Investigaciones recientes demuestran que su expansión geográfica es aún activa, en un área de conservación en la selva tropical atlántica en Brasil, en la región Sudeste. Loureiro et al., (2018) plantean que su presencia es alarmante y que la potencial simpatría entre $P$. clarkii y el cangrejo de río endémico del género Parastacus en la región Sudeste de Brasil, podría verse como una gran amenaza para el cangrejo de río nativo debido a la posibilidad de exclusión competitiva.

El modelo predice que las regiones montañosas de los Andes tienen condiciones ambientales que permiten la invasión por el cangrejo de río. Podría aparecer como idónea y no haber sido invadida. A pesar de estar presente en otras áreas andinas tendría alta probabilidad de expandirse e invadir esa zona. Se ha confirmado la presencia de $P$. clarkii en el lago Yahuarcocha perteneciente a la provincia de Imbabura de las montañas del norte de Ecuador. La especie no se ha expandido a los lagos vecinos Mojanda y San Pablo (Riascos et al., 2018). Es importante aclarar que su presencia en estas áreas es causa de la introducción humana con fines de acuicultura y expectativas sobre su uso en su producción, comercialización y mercado 
y no a la expansión natural a través de otras áreas invadidas (Mora et al., 2004).

En cuanto a la dispersión de $P$. clarkii en Colombia, esta especie se encuentra en cuerpos de agua que incluyen: estanques, lagos y zanjones. Las introducciones han sido realizadas en gran parte por el ser humano para fines de acuicultura, pesca y acuarismo (Campos, 2005). El modelo predijo que las áreas idóneas se ubican principalmente en el norte y oriente de Colombia, que incluyen diversos ecosistemas, como: bosques tropicales, bosques basales, bosques ribereños y sabanas. La especie tiene una alta posibilidad de expandirse hacia áreas de distribución de latitudes bajas, ocupando zonas hacia la parte norte de la región caribe colombiana, posiblemente, por la abundante oferta de recursos hídricos en la región, sus diversas lagunas y ciénagas inundados periódicamente por las crecidas de los ríos, en departamentos de: Magdalena, Cesar, Córdoba y Atlántico. También los pantanos en Bolívar y en Magdalena pueden ser ambientes idóneos para el posible establecimiento de la especie (Álvarez \& Polania, 1994).

Estudios de uso de hábitat han revelado que los cangrejos de río están adaptados para vivir en áreas que son alternadamente inundadas y secas (Huner \& Barr, 1984). La especie tiende a frecuentar aguas menos profundas y permanece cerca de las orillas, en lugar de habitar áreas a mayor distancia de la orilla a lagos, donde los lagos pueden alcanzar una profundidad máxima $(7.7 \mathrm{~m})$ y pantanos menos profundos $(<0.70 \mathrm{~m})$. Se demostró que el pantano, un sitio con menos profundidad mostró mejores condiciones que el lago para el crecimiento de individuos de cangrejos de río (Maccarrone et al., 2016; Donato et al., 2018). De hecho, profundidades en lagos mayores a 9 m no proporcionan a $P$. clarkii ciertas características ambientales que requieren (disponibilidad de alimentos, oportunidad de refugiarse y/o cavar madrigueras) (Bonvillain, Rutherford, Kelso, \& Green, 2012).

También se predijo áreas con idoneidad ambiental en el oriente de Colombia, probablemente la presencia de grandes extensiones de ecosistemas de sabanas en la región de la Orinoquia, con presencia de zonas inundables, que permiten el desarrollo de vegetación flotante, proporcionan ecosistemas temporales idóneos para el establecimiento de poblaciones sumideros de $P$. clarkii. Según estudio realizado por Vera-Ospina (2017), la Orinoquia es una región rica y diversa en plantas acuáticas, con 129 especies registradas en áreas de inundación como sabanas y humedales. La presencia de Eichornia crassipes en esta región podría ser un recurso valioso para $P$. clarkii, que puede usar como alimento, cubierta a depredadores (aves y peces), refugio y lugar donde capturar oxígeno en horas del día y la noche (Pedroza-Martínez, 2017). Se han realizado investigaciones que confirman que $P$. clarkii muestra preferencia por sitios con presencia de vegetación flotante causando impactos negativos en la reducción de la abundancia de macrófitas por pastoreo en los ecosistemas invadidos (Correia \& Anastácio, 2008; Cruz, Segurado, Sousa, \& Rebelo, 2008). La expansión de cultivos de arroz presente en las zonas de los Llanos Orientales que incluye: Meta, Casanare y Arauca (Fedearroz, 2011), brindaría a la especie el hábitat adecuado para que se estableciese en esa zona, ya que se ha registrado que una vez que la especie llega a una nueva localidad, puede causar daños y pérdidas económicas a las actividades agrícolas en áreas inundadas como las plantaciones de arroz (Correia \& Ferreira, 1995; Anastácio, Frias, \& Marques, 2000; Gherardi \& Barbaresi, 2000; Correia, 2002).

En el departamento del Valle del Cauca se ha registrado la presencia de esta especie; sin embargo, en el modelo se muestra como área de omisión. Este resultado posiblemente está asociado a los tipos de ecosistemas donde se reportó la especie, clasificados como zanjones, canales artificiales o canales de drenaje de cultivos, los cuales no son ecosistemas naturales (Flórez-Brand \& Espinosa-Beltrán, 2011). Por lo tanto, su presencia no ha representado un riesgo en ese lugar, debido a que no se encuentran especies nativas con las que $P$. clarkii pudiera competir por espacio o recursos y por ende desplazarlas para ocupar su nicho 
(Flórez-Brand \& Espinosa-Beltrán, 2011). Por otro lado, en la región del Pacifico colombiano se presentan condiciones de alta precipitación, temperatura y humedad, característico de un clima tropical húmedo de selva, con temperaturas mayores de $24{ }^{\circ} \mathrm{C}$ todo el año y lluvias permanentes (Pabón, Eslava, \& Goméz, 2001), lo que posibilita la ausencia de espacios ambientales idóneos para la presencia del cangrejo de rio $P$. clarkii.

El modelo predijo idoneidad ambiental en algunas áreas de Boyacá y Cundinamarca, ubicados en la región Andina que permiten la invasión por el cangrejo de río. En el 2004 ya se registraba para la sabana de Bogotá (Campos, 2005) y en 2008 en la laguna Fúquene y el área de influencia del río Suárez (Gutiérrez et al., 2012, Pachón \& Valderrama, 2018). AriasPineda y Rodríguez (2012), y Arias-Pineda y Pedroza-Martínez (2018) dan a conocer registros de la expansión de $P$. clarkii para Bogotá relacionado con actividades humanas en cinco localidades, entre ellas los humedales Juan Amarillo y Jaboque por liberación accidental. Se concluye que hay estabilidad poblacional de los cangrejos de río, indicando que se reproducen de manera exitosa, con poblaciones en aumento. Esta es una alerta a las autoridades ambientales a plantear estrategias de control para su establecimiento en el tiempo.

Estudios como el de Donato et al., (2018) destacan la importancia de la detección temprana en sitios que son potencialmente adecuados para la invasión de $P$. clarkii. Por lo que es importante sensibilizar a las poblaciones locales sobre el potencial impacto a mediano y a largo plazo de la presencia de esta especie en ecosistemas naturales. Esto con el fin de apoyar en reducir la propagación causada por la liberación de los individuos en entornos naturales. Adicionalmente, la erradicación del cangrejo de río es poco probable de conseguir por presentar plasticidad ecológica, tasa de crecimiento poblacional alta, cuidado parental que evita una mortalidad menor en su estadio larval como en otros decápodos, adaptación y tolerancia ambiental a condiciones nuevas de diferentes rangos térmicos, que le permite ocupar nuevos ambientes disponibles. Sugiriendo la importancia de monitorear la capacidad de dispersión y limitar la invasión de $P$. clarkii (Aquiloni, Ilhéu, \& Gherardi, 2005; Nunes, Hoffman, Zengeya, Measey, \& Weyl, 2017).

Con el análisis realizado, se brindó información actual de la especie y variables ambientales asociadas a su distribución, que permita apoyar con medidas de prevención y conservación para las especies nativas. Proponemos que las áreas identificadas como potencialmente idóneas principalmente las detectadas en la región Caribe, Orinoquia y región Andina deban ser monitoreadas para la presencia del cangrejo de río y así prevenir su introducción en áreas cercanas. Este estudio proporciona información de interés para construir una estrategia útil o un plan de manejo de la especie.

Declaración de ética: los autores declaran que todos están de acuerdo con esta publicación y que han hecho aportes que justifican su autoría; que no hay conflicto de interés de ningún tipo; y que han cumplido con todos los requisitos y procedimientos éticos y legales pertinentes. Todas las fuentes de financiamiento se detallan plena y claramente en la sección de agradecimientos. El respectivo documento legal firmado se encuentra en los archivos de la revista.

\section{AGRADECIMIENTOS}

Las ideas presentadas en este manuscrito son producto de un análisis del proceso de elaboración del trabajo de grado para obtener la titulación en biología. Los autores agradecen al programa de Biología de la Universidad del Quindío por el apoyo y la colaboración y a Martha Campos por su ayuda en la definición de la especie y sus consejos.

\section{RESUMEN}

Introducción: El proceso de invasión biológica es una de las mayores amenazas a la biodiversidad y ecosistemas, con actuales y potenciales impactos en la salud pública y conservación. Procambarus clarkii es un crustáceo 
decápodo, originario del sur de Estados Unidos y noreste de México, que puede adaptarse a diferentes condiciones ambientales debido a su plasticidad ecológica. Objetivo: Se caracterizó el nicho ecológico fundamental existente de Procambarus clarkii con el fin de predecir las áreas con idoneidad ambiental para el potencial establecimiento de la especie en Suramérica y Colombia. Métodos: Usamos modelos de nichos ecológicos calibrados en el área nativa, elaborados con el algoritmo Maxent, basados en datos de presencia extraídos de GBIF y variables hidroclimáticas de ecosistemas acuáticos a una resolución de $1 \mathrm{~km}^{2}$. Resultados: En Suramérica el modelo indicó un potencial geográfico amplio de invasión mostrando áreas ambientalmente idóneas para la presencia y expansión hacia Colombia, Venezuela, Perú, Ecuador, Brasil, Guyana, Surinam, Bolivia, Uruguay, Argentina, Paraguay y Chile. En Colombia, el modelo predijo que las áreas idóneas se ubican principalmente en el norte y oriente, incluidos diversos ecosistemas, como: bosques tropicales, bosques basales, bosques riparios y sabanas. La especie tiene una alta posibilidad de expandirse hacia áreas de distribución de latitudes bajas, ocupando zonas hacia la parte norte de la región Caribe colombiana, en departamentos de Magdalena, Cesar, Córdoba y Atlántico. También se predijo áreas con idoneidad ambiental en el oriente de Colombia, hacia la extensión de la planicie oriental de la Orinoquia, una región de baja altitud en: Arauca, Casanare, Meta y Vichada. Conclusión: En este estudio se aplican modelos de nichos ecológicos, que puede ser de interés en la planeación de estrategias o la creación de planes de manejo, como sistemas de alerta temprana para evitar el establecimiento de esta especie.

Palabras clave: cangrejo rojo de pantano; manejo de especies exóticas; nicho ecológico; distribución potencial.

\section{REFERENCIAS}

Alcorlo, P., Otero, M., Crehuet, M., Baltanás, A., \& Montes, C. (2006). The use of the red swamp crayfish (Procambarus clarkii, Girard) as indicator of the bioavailability of heavy metals in environmental monitoring in the River Guadiamar (SW, Spain). Science of the Total Environment, 366(1), 380-390.

Álvarez-León, R., \& Polanía. J. (1994). Manglares, lagunas costeras y estuarios del caribe colombiano. Memorias del taller de expertos sobre el estado de conocimiento y lineamientos para una estrategia nacional de biodiversidad en los ecosistemas marinos y costeros, Minca-Magdalena. Bogotá, Colombia: Colciencias.

Anastácio, P.M., Frias, A.F., \& Marques, J.C. (2000). Impact of crayfish densities on wet seeded rice and the inefficiency of a non-ionic surfactant as an ecotechnological solution. Ecological Engineering, $15,17-25$.
Aquiloni, L., Ilhéu, M., \& Gherardi, F. (2005). Habitat use and dispersal of the invasive crayfish Procambarus clarkii in ephemeral water bodies of Portugal. Marine and Freshwater Behaviour and Physiology, 38(4), 225-236.

Aquiloni, L., Martín, M.P., Gherardi, F., \& DiéguezUribeondo, J. (2011). The North American crayfish Procambarus clarkii is the carrier of the oomycete Aphanomyces astacy in Italy. Springer, 13, 359-367.

Arias-Pineda, J.Y., \& Rodríguez, W.D. (2012). First record of the invasive species Procambarus (Scapulicambarus) clarkii (Girard 1852) (Crustacea, Decapoda, Cambaridae) from the Colombian Eastern Cordillera. Boletín de La Sociedad Entomológica Aragonesa, 51, 313-315.

Arias-Pineda, J.Y., \& Pedroza-Martínez, D.V. (2018). Presencia del cangrejo rojo Procambarus clarkii (Girard, 1852) en la sabana de Bogotá, Colombia. Boletín de la Sociedad Entomológica Aragonesa (S.E.A), 62, 283-286.

Atkinson, R.J., \& Taylor, A.C. (1988). Physiological ecology of burrowing decapods. Symposium Zoology. Society London, 59, 201-226.

Baigún, R. (2001). Ecología pesquera de lagos y embalses patagonicos (Argentina) (Tesis de doctorado). Universidad de Buenos Aires, Buenos Aires, Argentina.

Banci, K., Torello-Viera, N.F., Marinho, P.S., Calixto, P., \& Marques, O. (2013). Predation of Rhinella ornata (Anura, Bufonidae) by the alien crayfish (Crustacea, Astacidae) Procambarus clarkii (Girard, 1852) in São Paulo, Brazil. Herpetology Notes, 6(1), 339-341.

Barbaresi, S., Santini, G., Tricarico, E., \& Gherardi, F. (2004). Ranging behaviour of the invasive crayfish, Procambarus clarkii (Girard). Journal of Natural History, 38(22), 2821-2832.

Barve, N., Barve, V., Jiménez-Valverde, A., Lira-Noriega, A., Maher, S.P., Peterson, A.T., ... Villalobos, F. (2011). The crucial role of the accessible area in ecological niche modeling and species distribution modeling. Ecological Modelling, 222(11), 1810-1819.

Bonvillain, C.P., Rutherford, D.A., Kelso, W.E., \& Green, C.C. (2012). Physiological biomarkers of hypoxic stress in red swamp crayfish Procambarus clarkii from field and laboratory experiments. Comparative Biochemistry and Physiology - A Molecular and Integrative Physiology, 163(1), 15-21.

Campos, M. (2005). Procambarus (Scapulicambarus) clarkii (Girard, 1852), (Crustacea: Decapoda: Cambaridae). Una langostilla no nativa en Colombia. Revista de la Academia Colombiana de Ciencias Exactas, Fisicas y Naturales, 29(111), 295-302. 
Chien, Y.H., \& Avault, J.W. (1983). Effects of flooding dates and disposals of rice straw on crayfish, Procambarus clarkii (Girard), culture in rice fields. Aquaculture, 31(2), 339-359.

Chung, Y.S., Cooper, R.M., Graff, J., \& Cooper, R.L. (2012). The acute and chronic effect of low temperature on survival, heart rate and neural function in crayfish (Procambarus clarkii) and prawn (Macrobrachium rosenbergii) species. Open Journal of Molecular and Integrative Physiology, 2, 75-86.

Crandall, K.A. (2010). Procambarus clarkii. The IUCN Red List of Threatened Species 2010: an online reference. Recuperado de http://dx.doi.org/10.2305/ IUCN.UK.20103.RLTS.T153877A557336

Correia, A.M., \& Ferreira, O. (1995). Burrowing behavior of the introduced red swamp crayfish Procambarus clarkii (Decapoda: Cambaridae) in Portugal. Journal of Crustacean Biology, 15, 248-257.

Correia, A.M. (2002). Niche breadth and trophic diversity: Feeding behaviour of the red swamp crayfish (Procambarus clarkii) towards environmental availability of aquatic macroinvertebrates in a rice field (Portugal). Acta Oecologica, 23(6), 421-429.

Correia, A.M., \& Anastácio, P.M. (2008). Shifts in aquatic macroinvertebrate biodiversity associated with the presence and size of an alien crayfish. Ecological Research, 23, 729-734.

Cruz, M.J., \& Rebelo, R. (2005). Vulnerability of Southwest Iberian amphibians to an introduced crayfish, Procambarus clarkii. Amphibia Reptilia, 26(3), 293-303.

Cruz, M.J., Segurado, P., Sousa, M., \& Rebelo, R. (2008). Collapse of the amphibian community of the Paul do Boquilobo Natural Reserve (central Portugal) after the arrival of the exotic American crayfish Procambarus clarkii. Herpetological Journal, 18, 197-204.

De Pirro, M., Cannicci, S., \& Santini, G. (1999). A multifactorial experiment on heart rate variations in the intertidal crab Pachygrapsus marmoratus. Marine biology, 135, 341-345.

Domisch, S., Amatulli, G., \& Jetz, W. (2015). Near-global freshwater-specific environmental variables for biodiversity analyses in $1 \mathrm{~km}$ resolution. Scientific Data, $2,1-13$

Donato, R., Rollandin, M., Favaro, L., Ferrarese, A., Pessani, D., \& Ghia, D. (2018). Habitat use and population structure of the invasive red swamp crayfish Procambarus clarkii (Girard, 1852) in a protected area in Northern Italy. Knowledge and Management of Aquatic Ecosystems, 419, 1-12.

Elith, J. (2013). Predicting distributions of invasive species. Melbourne, Australia: University of Melbourne.
Eshky, A.A., Atkinson, R.J., \& Taylor, A.C. (1995). Physiological ecology of crabs from Saudi Arabian mangrove. Marine Ecology Progress Series, 126, 83-95.

Eshky, A.A., Taylor, A.C., \& Atkinson, R.J. (1996). The effects of temperature on aspects of respiratory physiology of the semi-terrestrial crabs, Uca inversa (Hofiftnann) and Metopograpsus messor (Forskal) from the Red Sea. Comparative Biochemistry and Physiology, 114A(4), 297-304.

Everett, R.A. (2000). Patterns and pathways of biological invasions. Trends in Ecology and Evolution, 15(5), 177-178.

Fedearroz. (2011). Dinámica del sector arrocero de los Llanos Orientales de Colombia, 1999-2011. Bogotá, Colombia: Federación Nacional de Arroceros.

Flórez-Brand, P., \& Espinosa-Beltrán, J. (2011). Presencia y dispersión del cangrejo rojo americano (Procambarus clarkii Girard, 1852) (Decapoda: Cambaridae) en el departamento del Valle del Cauca, Colombia. Biota Colombiana, 12(2), 57-62.

Franco, M.K. (2014). Morfometría, distribución actual y potencial en el norte de México del acocil rojo Procambarus clarkii (Girard, 1852) (Crustacea: Cambaridae) (Tesis de maestría). Universidad de Nuevo León, Monterrey, México.

Gál, B., Gábris, V., Cser, B., Danyik, T., Farkas, A., Farkas, J., ... Weiperth, A. (2018). Present distribution of the invasive red swamp crayfish Procambarus clarkii (Girard 1852) and its effects on the fish fauna assemblages in some tributaries of the Hungarian section of the River Danube. Pisces Hungarici, 12, 71-76.

Gallien, L., Münkemüller, T., Albert, C.H., Boulangeat, I., \& Thuiller, W. (2010). Predicting potential distributions of invasive species: Where to go from here? Diversity and Distributions, 16(3), 331-342.

GBIF. (2019). Global Biodiversity Information Facility. Free and open access to biodiversity data. Recuperado de https://www.gbif.org

Gherardi, F., \& Barbaresi, S. (2000). Invasive crayfish: activity patterns of Procambarus clarkii in the rice fields of the Lower Guadalquivir (Spain). Fundamental and Applied Limnology, 150(1), 153-168.

Gherardi, F. (2006). Crayfish invading Europe: The case study of Procambarus clarkii. Marine and Freshwater Behaviour and Physiology, 39(3), 175-191.

Gherardi, F., \& Acquistapace, P. (2007). Invasive crayfish in Europe: the impact of Procambarus clarkii on the littoral community of a Mediterranean lake. Freshwater Biology, 52(7), 1249-1259.

Gómez, E. (2014). Evaluación del valor nutricional y pigmentante de la harina de cangrejo Procambarus clarkii para la alimentación de gallinas semipesadas 
y pollos de engorde como método de control poblacional del cangrejo (Tesis de doctorado). Universidad Nacional de Colombia, Palmira, Colombia.

Gutiérrez, F.P., Lasso, C., Baptiste, M.P., Sánchez-Duarte, P., \& Díaz, A. (2012). Catálogo de la biodiversidad acuática exótica y trasplantada en Colombia: moluscos, crustáceos, peces, anfibios, reptiles y aves. Bogotá, Colombia: Instituto de Investigación de Recursos Biológicos Alexander von Humboldt.

Halwart, M., \& Gupta, M.V. (2004). Culture of Fish in Rice Fields. Penang, Malasia: WorldFish.

Hobbs, H.H. (1972). Biota of freshwater ecosystems. Identification manual No. 9. Crayfishes (Astacidae) of North and middle America. Smithsonian Contribution, Washington DC, Department of invertebrate Zoology, 184, 1-222.

Huang, J., Tang, S., Cai, F., Lin, Y., \& Wu, Z. (2017). Microsatellite evidence of dispersal mechanism of red swamp crayfish (Procambarus clarkii) in the Pearl River basin and implications for its management Scientific Reports, 7, 1-8.

Huner, J.V. (1977). Introductions of the Louisiana red swamp crayfish, Procambarus clarkii (Girard): an update. Freshwater Crayfish, 3, 193-202.

Huner, J.V., \& Avault, J.W. (1979). Introductions of Procambarus spp. Freshwater Crayfish, 4, 191-194.

Huner, J.V., \& Barr, E. (1984). Red swamp crayfish: biology and exploitation. Louisiana sea grant program, center for wetland resources. Baton Rouge, Estados Unidos de América: Louisiana State University.

Huner, J.V. (2002). Procambarus. In D.M. Holdich (Ed.), Biology of freshwater crayfish (pp. 541-584). Reino Unido: Blackwell Scientific Press.

Lizarralde, M.S. (1993). Current status of the introduced beaver (Castor canadensis) population in Tierra del Fuego, Argentina. Ambio, 22(6), 351-358.

Loureiro, T.G., Anastácio, P.M., Araujo, P.B., Souty-Grosset, K., \& Almerão, M.P. (2015). Red swamp crayfish: biology, ecology and invasion - an overview. Nauplius (Sociedade Brasileira de Carcinologia), 23(1), 1-19.

Loureiro, T.G., Anastácio, P.M., Bueno, S.L. de S., \& Araujo, P.B. (2018). Management of invasive populations of the freshwater crayfish Procambarus clarkii (Decapoda, Cambaridae): test of a population-control method and proposal of a standard monitoring approach. Environmental Monitoring and Assessment, 190(9), 559-569.

Maccarone, V., Filiciotto, F., Buffa, G., Di Stefano, V., Quinci, E.M., Vicenzi, G., ... Buscaino, G. (2016). An invasive species in a protected area of Southern Italy: the structure, dynamics and spatial distribution of the crayfish Procambarus clarkii. Turkish Journal of Fisheries and Aquatic Sciencies, 16, 401-412.

Magalhães, C., Bueno, S.L.S., Bond-Buckup, G., Valenti, W.C., Melo Da Silva, H.L., Kiyohara, F., \& Rocha, S.S. (2005). Exotic species of freshwater decapod crustaceans in the state of São Paulo, Brazil: Records and possible causes of their introduction. Biodiversity and Conservation, 14(8), 1929-1945.

Mora, V., Uyuguari, M., \& Osorio, V. (2004). Situación actual de las especies introducidas en el Ecuador con fines acuícolas. Guayaquil, Ecuador: Escuela Superior Politécnica del Litoral.

Muscarella, R., Galante, P.J., Soley-Guardia, M., Boria, R.A., Kass, J.M., Uriarte, M., \& Anderson, R.P. (2014). ENMeval: An R package for conducting spatially independent evaluations and estimating optimal model complexity for Maxent ecological niche models. Methods in Ecology and Evolution, 5(11), 1198-1205.

Nunes, A.L., Hoffman, A.C., Zengeya, T.A., Measey, G.J., \& Weyl, O.L. (2017). Red swamp crayfish, Procambarus clarkii, found in South Africa 22 years after attempted eradication. Aquatic Conservation: Marine and Freshwater Ecosystems, 27(6), 1334-1340.

Pabón, J., Eslava, J., \& Gómez, R. (2001). Generalidades de la distribución espacial y temporal de temperatura del aire y de la precipitación en Colombia. Meteorología Colombiana, 4, 47-59.

Pachón, Y., \& Valderrama, B. (2018). Anotaciones al estado, uso y gestión de la langostilla roja Procambarus (Scapulicambarus) clarkii, una especie invasora en la laguna de Fúquene (Cundinamarca, Colombia). Biodiversidad en la práctica, 3(1), 30-51.

Palaoro, A.V., Dalosto, M.M., Costa, G.C., \& Santos, S. (2013). Niche conservatism and the potential for the crayfish Procambarus clarkii to invade South America. Freshwater Biology, 58(7), 1379-1391.

Pearson, R.G., Raxworthy, C.J., Nakamura, M., \& Peterson, A.T. (2007). Predicting species distributions from small numbers of occurrence records: A test case using cryptic geckos in Madagascar. Journal of Biogeography, 34(1), 102-117.

Pedroza-Martínez, D.R. (2017). Estudio de la estructura poblacional de Procambarus clarkii (Girard, 1852), en la Laguna de Fúquene, Cundinamarca (Tesis de pregrado). Universidad Distrital Francisco José de Caldas, Colombia

Peterson, A.T. (2003). Predicting the geography of species' invasions via ecological niche modeling. The Quarterly Review of Biology, 78(4), 419-433.

Peterson, A.T., Papeş, M., \& Soberón, J. (2008). Rethinking receiver operating characteristic analysis applications 
in ecological niche modeling. Ecological Modelling, 213(1), 63-72.

Peterson, A.T., Soberón, J., Pearson, R.G., Anderson, R.P., Martínez-Meyer, E., Nakamura, M., \& Araújo, M.B. (2011). Ecological niches and geographic distributions. Nueva Jersey, Estados Unidos de América: Princeton University Press.

Peterson, A.T. (2014). Mapping disease transmission risk. Baltimore, Estados Unidos de América: Jhons Hopkins University Press.

Pimentel, D., Lach, L., Zuniga, R., \& Morrison, D. (2000). Environmental and economic costs associated non nonindigenous species in the United States. Bioscience, 50(1), 53-65.

Pourrut, P. (1983). Los climas del Ecuador: fundamentos explicativos. Quito, Ecuador: Centro Ecuatoriano de Investigaciones Geográficas.

R Development Core Team. (2014). $R$ a language and environment for statistical computing. R Foundation for Statistical Computing, Vienna, Austria. Recuperado de http//www.R-project.org

Riascos, L., Geerts, A.N., Oña, T., Goethals, P., CevallosCevallos, J., Vandenberghe, W., ... Van der heyden, C. (2018). DNA-based monitoring of the alien invasive North American crayfish Procambarus clarkii in Andean lakes (Ecuador). Limnologica, 70, 20-25.

Rodríguez, G., \& Suárez, H. (2001). Anthropogenic dispersal of Decapod Crustaceans in aquatic environment. Interciencia, 26(7), 282-288.

Romero-Trigueros, L.E. (1988). Reproducción y desarrollo post-larval del camarón de río Procambarus clarkii (Girard,1852) (Tesis profesional). Universidad del Valle, Colombia.

Sakai, A.K., Allendorf, F.W., Holt, J.S., Lodge, M., Molofsky, J., With, K.A., ... Weller, S.G. (2001). The population biology of invasive species. Annual Review of Ecology and Systematics, 32, 305-332.

Servicio de Información Ambiental y Geográfica, (SIAG) DEL Centro Austral de Investigaciones Científicas. Recuperado de www.cadic.gov.ar, SIAG

Schiavini, A., Carranza, M.L., Deferrari, G., Escobar, J., Malmierca, L., \& Pietrek, A.G. (2016). Erradicación de especies invasoras: ciencia, actitud y entendimiento. El castor en Tierra del Fuego. Mastozoología Neotropical, 23(2), 279-288.
Simberloff, D., Parker, I.M., \& Windle, P.N. (2005). Introduced species policy, management, and future research needs. Frontiers in Ecology and the Environment, 3(1), 12-20.

Sommer, T.R., (1984). The biological response of the crayfish Procambarus clarkii to transplantation into California ricefields. Aquaculture, 41(4), 373-384.

Sousa, R., Morais, P., Dias, E., \& Antunes, C. (2011). Biological invasions and ecosystem functioning: time to merge. Biological Invasions, 13(5), 1055-1058.

Souty-Grosset, C., Anastácio, P.M., Aquiloni, L., Banha, F., Choquer, J., Chucholl, C., \& Tricarico, E. (2016). The red swamp crayfish Procambarus clarkii in Europe: Impacts on aquatic ecosystems and human well-being. Limnologica, 58, 78-93.

Stillman, J.H., \& Somero, G.N. (1996). Adaptation to temperature stress and aerial exposure in congeneric species of intertidal porcelain crabs (Genus Petrolisthes): correlation of physiology, biochemistry and morphology with vertical distribution. Journal of Experimental Biology, 199, 1845-1855.

Thuiller, W., Richardson, D.M., Pysek, P., Midgley, G.F., Greg, O., \& Rouget, H.M. (2005). Niche-based modelling as a tool for predicting the risk of alien plant invasions at a global scale. Global Change Biology, 11, 2234-2250.

Torres, E., \& Álvarez, F. (2012). Genetic variation in native and introduced populations of the red swamp crayfish Procambarus clarkii (Girard, 1852) (Crustacea, Decapoda, Cambaridae) in Mexico and Costa Rica. Aquatic Invasions, 7(2), 235-241.

Vera-Ospina, A. (2017). Flora y vegetación acuática en áreas de la Orinoquia Colombiana (Tesis de Maestria). Universidad Nacional de Colombia, Colombia.

Vitousek, P.M., D’Antonio, C.M., Lloyd, L.L., \& Westbrooks, R. (1996). Biological invasions as global environmental change. American Scientist, 84(5), 218-228.

West, A.M., Kumar, S., Brown, C.S., Stohlgren, T.J., \& Bromberg, J. (2016). Field validation of an invasive species Maxent model. Ecological Informatics, 36, 126-134.

Yi, S., Li, Y., Shi, L., Zhang, L., Li, Q., \& Chen, J. (2018). Characterization of Population Genetic Structure of red swamp crayfish, Procambarus clarkii, in China. Scientific Reports, 8, 1-11.

See Digital Appendix at: / Ver Apéndice digital en: revistas.ucr.ac.cr 